\title{
Global attractor of a differentiable autonomous system on the plane
}

\author{
by NGuyen Van Chau (Hanoi)
}

\begin{abstract}
We study the structure of a differentiable autonomous system on the plane with non-positive divergence outside a bounded set. It is shown that under certain conditions such a system has a global attractor. The main result here can be seen as an improvement of the results of Olech and Meisters in [7, 9] concerning the global asymptotic stability conjecture of Markus and Yamabe and the Jacobian Conjecture.
\end{abstract}

1. Introduction. Consider the autonomous system

$$
\dot{x}=f(x)
$$

on $\mathbb{R}^{2}$, where $f$ is $C^{1}$ on $\mathbb{R}^{2}$. Let $D f(x)$ and $\operatorname{Div} f(x)$ denote the Jacobian matrix and the divergence of $f$.

In 1962 C. Olech [7] considered the 2-dimensional case of the MarkusYamabe Conjecture [5] on the global asymptotic stability of autonomous systems and obtained the following result:

Suppose that

(i) $f(0)=0$,

(ii) det $D f(x)>0$ and $\operatorname{Div} f(x)<0$ for all $x \in \mathbb{R}^{2}$, and

(iii) $\|f(x)\|>$ const $>0$ for $\|x\|>$ const.

Then $x=0$ is a globally asymptotically stable solution of the system $(\mathrm{S})$.

This result leads to an affirmative solution of the global asymptotic stability conjecture of Markus and Yamabe for the 2-dimensional polynomial case [9], that the conditions (i) and (ii) imply global asymptotic stability of the solution $x=0$.

1991 Mathematics Subject Classification: 34D05, 58F10, 58F12, 58F21.

Key words and phrases: Markus-Yamabe Conjecture, asymptotically stable, Jacobian Conjecture. 
In this note we present an improvement of these results for the case when the above condition for Div $f$ only holds outside a bounded set. Our main result is the following.

THEOREM 1. Suppose that

(i) $f(0)=0$ and zero is a regular value of $f$,

(ii) $\|f(x)\|>$ const $>0$ for $\|x\|>$ const,

(iii) $\operatorname{Div} f(x) \leq 0$ for $\|x\|>$ const, and

(iv) $\int_{\mathbb{R}^{2}} \operatorname{Div} f(x) d x<0$.

Then either

(a) there is a trajectory with empty positive limit set which tends to a saddle point as $t \rightarrow-\infty$, or

(b) the system (S) has a global attractor.

In fact, it will be shown that under conditions (i)-(iii) either there exists a special trajectory as in (a), or infinity can be viewed as either a centre, a repulsive point or an attracting point of the system. It seems that the last case cannot happen. This will be proved for the polynomial case.

A special case of these results is when the system has no saddle point. For this case, it will be shown that under conditions (i)-(iii) the system has a unique singular point. This fact allows us to obtain some results concerning the univalence domain of a map. In particular, one of these results asserts that a polynomial map of $\mathbb{R}^{2}$ into itself with $\operatorname{det} D f$ positive is a diffeomorphism of $\mathbb{R}^{2}$ if $\operatorname{Div} f(x)$ is non-positive outside a bounded set. This is an improvement of a result of Olech and Meisters [9] concerning the real version of the Jacobian Conjecture, which asserts that every polynomial non-singular map of $\mathbb{R}^{n}$ into itself is bijective.

The proof of Theorem 1 is based on Olech's Lemma about orbital stability of plane dynamical systems in invariant domains of non-positive divergence [7, 8]. This lemma and some other lemmas are given in Section 2. Theorem 1 and its version for the polynomial case are proved in Section 3. Some results on univalence are presented in the last section.

The author would like to take this opportunity to thank Prof. C. Olech and Prof. M. Zelikin for advice and support. The author also wishes to thank the International Centre for Theoretical Physics, Trieste, and the Banach International Mathematical Center, Warsaw, for their hospitality.

2. Some lemmas. Let $f$ be a $C^{1}$-map of $\mathbb{R}^{2}$ into itself, $f=\left(f_{1}, f_{2}\right)$. We consider the autonomous system

$$
\dot{x}=f(x)
$$


and its orthogonal system

$$
\dot{x}=\left(-f_{2}(x), f_{1}(x)\right) .
$$

Denote by $x(t, P)\left(\right.$ resp. $\left.x^{*}(t, P)\right)$ the unique solution of $(\mathrm{S})\left(\right.$ resp. $\left.\left(\mathrm{S}^{*}\right)\right)$ with the initial condition $x(0, P)=P\left(\right.$ resp. $\left.x^{*}(0, P)=P\right)$. Let $(a(P), b(P))$ be the maximal interval on which $x(t, P)$ is defined. As usual, $\omega(P)$ and $\alpha(P)$ denote the positive and negative limit sets of $x(t, P)$. Denote by $P_{1} P_{2}$ the segment $\left\{x=x(t, P): t_{2} \leq t \leq t_{1}\right\}$, where $P_{i}=x\left(t_{i}, P\right), i=1,2$.

For the solution segment $P Q$ of $\left(\mathrm{S}^{*}\right), Q=x^{*}\left(t_{0}, P\right)$, Olech $[7,8]$ introduced the function

$$
L(P, Q)=\int_{P Q}\left\|f\left(x^{*}(t, P)\right)\right\|^{2} d t=\int_{0}^{s_{0}}\left\|f\left(x^{*}(t(s), P)\right)\right\| d s,
$$

where $d s=\left\|f\left(x^{*}(t, P)\right)\right\| d t$ is the line element and $s_{0}$ is the length of the segment $P Q$. Put $P(t)=x(t, P)$. There is an $\varepsilon>0$ such that for each $t \in$ $[0, \varepsilon)$ the solution curve $x^{*}(\tau, P(t))$ crosses the solution curve $x(t, Q)$ at time $\tau(t)>0$. By the continuous dependence of solutions on initial conditions, there exists an increasing continuous function $s(t)$ and a continuous function $\tau(t)$ on $[0, \varepsilon)$ satisfying $\tau(0)=0$ and $s(0)=t_{0}$ such that $x(s(t), Q)=$ $x^{*}(\tau(t), P(t))$.

Assume that these functions are well defined on the maximal interval $[0, T(P))$. Put $Q(t)=x(s(t), Q)$. We denote by $G(t)$ the region with boundary $P P(t) Q(t) Q$, where $P P(t), Q Q(t)$ and $P Q, P(t) Q(t)$ are solution segments of $(\mathrm{S})$ and $\left(\mathrm{S}^{*}\right)$, respectively. Using Green's formula we obtain

$$
L(P, Q)-L(P(t), Q(t))=-\int_{G(t)} \operatorname{Div} f(x) d x .
$$

For $\mu>0$, put $O(P, \mu):=\bigcup_{t>0}\{x:\|x-x(t, P)\|<\mu\}$. This is a neighbourhood of the solution curve $x(t, P), t \geq 0$.

The following lemma, for convenience restated in another form, is due to Olech $[7,8]$.

Olech's Lemma (see Lemma 2 in [7]). Assume that there are constants $\mu>0$ and $d>0$ such that $\|f(x)\|>d$, Div $f(x) \leq 0$ for $x \in O(P, \mu)$, $P Q \subset O(P, \mu)$ and $L(P, Q)<\mu d$. Then

(i) $T(P)=b(P)$,

(ii) $s(t) \rightarrow b(Q)$ as $t \rightarrow b(P)$,

(iii) $d\|P(t)-Q(t)\| \leq L(P(t), Q(t)) \leq L(P, Q) \leq \mu d$ for all $0 \leq t<b(P)$.

Denote by $A_{\infty}$ the set of all points $P$ such that $\omega(P)=\emptyset$. Applying Olech's Lemma to the case when $\operatorname{Div} f$ is non-positive outside a bounded set we have 
LEMma 1. Assume that there are $d>0$ and $r>0$ such that

$$
\|f(x)\|>d \quad \text { and } \operatorname{Div} f(x) \leq 0 \quad \text { for }\|x\|>r .
$$

Then

(i) the set $A_{\infty}$ is open,

(ii) for any $P \in \mathbb{R}^{2}$ either $\omega(P)=\emptyset$ or $\omega(P)$ is bounded.

P r o of. (i) follows immediately from Olech's Lemma. If $\omega(P) \neq \emptyset$ were unbounded then in view of the Poincaré-Bendixson Theorem it would contain a trajectory with empty positive limit set. Hence, by $(\mathrm{i}), \omega(P)=\emptyset$, which contradicts the unboundedness of $\omega(P)$. Thus $\omega(P)$ is bounded.

Remark 1. In the case when (3) holds, for each $P \in A_{\infty}$ and each sufficiently small solution segment $P Q$ of $\left(\mathrm{S}^{*}\right)$ the functions $P(t), Q(t)$ and $G(t)$ are well defined and the properties (i) and (ii) in Olech's Lemma are still valid. Instead of property (iii) we have

(iii) $^{\prime} d\|P(t)-Q(t)\| \leq L(P(t), Q(t)) \leq L\left(P\left(t_{0}\right), Q\left(t_{0}\right)\right)$

for $0 \leq t_{0} \leq t \leq b(P)$. Furthermore,

$$
L(P, Q)=-\int_{G_{P Q}} \operatorname{Div} f(x) d x+\lim _{t \rightarrow b(P)} L(P(t), Q(t)),
$$

where $G_{P Q}=\bigcup_{t>0} G(t)$.

From Olech's Lemma we can observe an interesting geometrical relation between the trajectories of the systems $(\mathrm{S})$ and $\left(\mathrm{S}^{*}\right)$.

Lemma 2. Let $L$ be a trajectory of $\left(\mathrm{S}^{*}\right)$. For each $P \in A_{\infty} \cap L$ the solution curve $x(t, P), t>0$, either does not cross $L$ again, or it crosses $L$ infinitely many times.

Proof. Let $V$ be a connected component of $A_{\infty} \cap L$ and $P \in V$. As in Remark 1 we can choose a sufficiently small solution segment $P_{1} P_{2}$ with $P \in P_{1} P_{2}$ so that the corresponding functions $P_{1}(t)$ and $P_{2}(t)$ are well defined and satisfy (i), (ii) and (iii) ${ }^{\prime}$ of Remark 1 . Clearly, the curves $P_{i}(t)$, $t \geq 0$, cross $V$ iff the curve $x(t, P), t \geq 0$, crosses $V$. Since $V$ is connected, this implies that the set

$$
W:=\{P \in V: x(t, P) \in V \text { for some } t>0\}
$$

is either empty or the whole of $V$. In the latter case, if $P \in W$ then the curve $x(t, P), t \geq 0$, crosses $V$ infinitely many times, which completes the proof.

3. Global attractor. In this section we give the proof of Theorem 1 and a version for the polynomial case. Theorem 1 will be proved in a sequence of lemmas. 
Consider the system $(\mathrm{S})$ under the assumptions of Theorem 1:

$$
\begin{aligned}
& f(0)=0 \quad \text { and } \quad \operatorname{det} D f(x) \neq 0 \quad \text { for } x \in f^{-1}(0), \\
& \operatorname{Div} f(x) \leq 0 \text { for }\|x\|>r \text {, } \\
& \|f(x)\|>d \quad \text { for }\|x\|>r
\end{aligned}
$$

and

$$
\int_{\mathbb{R}^{2}} \operatorname{Div} f(x) d x<0
$$

where $r$ and $d$ are positive numbers.

Note that (7) and (9) mean that (S) has a finite number of singular points and its singular points are either attracting, repulsive, saddle points or centres (see e.g. [1]).

Lemma 3. Assume that the conditions (7)-(9) hold. Then either

(a) the set $A_{\infty}$ is not empty,

(b) the system (S) has a global attractor, or

(c) every trajectory of (S) starting from outside a bounded set is closed.

Pro of. Assume that $A_{\infty}=\emptyset$. Then, by Lemma 1 , for every $P \in \mathbb{R}^{2}$ the set $\omega(P)$ is bounded. Set

$$
B:=\{\omega(P): P \notin \omega(P)\} \quad \text { and } \quad C:=\bigcup_{B} \omega(P) .
$$

First, we show that $C$ is bounded. Notice that by (7) all elements of $B$ are regular trajectories, except finitely many elements which are either singular points, or solution curves composed by some singular points and some trajectories joining singular points. Clearly, if the family $B$ is finite, then $C$ is bounded. Consider the case when $B$ is infinite. Assume, contrary to our claim, that $C$ is unbounded. Then we can define a sequence $c_{i} \in B$, $i=1,2, \ldots$, such that $D_{i} \subset D_{i+1}$ and

$$
K:=\bigcup_{i=1}^{\infty} c_{i} \text { is unbounded, }
$$

where $D_{i}$ denotes the bounded component of $\mathbb{R}^{2} \backslash c_{i}$. Notice that by (8),

$$
c_{i} \cap\{x:\|x\| \leq r\} \neq \emptyset .
$$

Since $K$ is unbounded, from (34) it follows that there are sequences $P_{i_{k}}$ and $t_{i_{k}}>0$ such that $P_{i_{k}} \in c_{i_{k}},\left\|P_{i_{k}}\right\|=r,\left\|x\left(t, P_{i_{k}}\right)\right\|>r$ for $0<t \leq t_{i_{k}}$, and

$$
\lim _{k \rightarrow \infty} P_{i_{k}}=P, \quad \lim _{k \rightarrow \infty} t_{i_{k}}=T, \quad \lim _{k \rightarrow \infty}\left\|x\left(t_{i_{k}}, P_{i_{k}}\right)\right\|=\infty .
$$


By the continuous dependence of solutions on initial conditions the solution $x(t, P)$ is well defined for $t<T$ and

$$
\lim _{t \rightarrow T}\|x(t, P)\|=\infty .
$$

This means that $\omega(P)=\emptyset$, contrary to the assumption $A_{\infty}=\emptyset$. Thus, we have proved that $C$ is bounded.

Now, denote by $E$ the unbounded component of $\mathbb{R}^{2} \backslash \operatorname{cl}(C)$. Then, for each $p \in E$ either $x(t, P)$ is a closed trajectory or $\omega(P) \subset \operatorname{cl}(C)$. Put

$$
F:=\{P \in E: P \in \omega(P)\} .
$$

Notice that every trajectory starting from $F$ is closed, and $F \cup\left(\mathbb{R}^{2} \backslash E\right)$ is a global attractor of $(\mathrm{S})$ if $F$ is bounded. So, to complete the proof we need only show that if $F$ is not a neighbourhood of infinity then $F$ is bounded. Assuming the unboundedness of $F$, in the same way as above we deduce that $A_{\infty} \neq \emptyset$, which is a contradiction. Thus $F$ is bounded.

Lemma 4. Assume that the conditions (7)-(9) hold and $A_{\infty} \neq \emptyset$. Then either

(a) there is a point $P$ such that $\omega(P)=\emptyset$ and $\alpha(P)$ is a saddle point, or

(b) the boundary $\mathrm{Bd} A_{\infty}$ of $A_{\infty}$ is either a repulsive point, a cycle or a polycycle and $\mathrm{Bd} A_{\infty}=\alpha(P)$ for all $P \in A_{\infty}$.

Proof. Assume that there is no point $P$ such that $\omega(P)=\emptyset$ and $\alpha(P)$ is a saddle point. We need to show that case $(\mathrm{b})$ happens.

First, we prove that $\mathrm{Bd} A_{\infty}$ is bounded. Notice that $\mathrm{Bd} A_{\infty}$ is an invariant closed set composed by solutions of $(\mathrm{S})$. Assume to the contrary that $\mathrm{Bd} A_{\infty}$ is unbounded. Then there is a $P \in \mathrm{Bd} A_{\infty}$ such that the trajectory $M:=\{x(t, P): t \in \mathbb{R}\}$ is unbounded. We can define a sequence $Q_{i} \in A_{\infty}$ such that $\left\|Q_{i}\right\|>r$, and $Q_{i}$ tends to $Q \in M$ as $i \rightarrow \infty$. Put

$$
L:=\left\{S=\lim x\left(t_{i}, Q_{i}\right): t_{i}>0\right\} .
$$

Then $L$ is a connected unbounded set composed by some solution of $(\mathrm{S})$ and we have $L \cap A_{\infty} \neq \emptyset$. In particular, $x(t, Q) \in L$ for all $t>0$. Let $Q^{*} \in L \cap A_{\infty}$. Then, clearly, either $\alpha\left(Q^{*}\right)$ is a saddle point or $Q^{*}=x(t, Q)$ for some $t$. Since neither of these cases can happen under our assumptions, it follows that $\mathrm{Bd} A_{\infty}$ is bounded.

Now, we prove that $\mathrm{Bd} A_{\infty}$ is connected. Let $C$ be a connected component of $\mathrm{Bd} A_{\infty}$. We first show that there is an open neighbourhood $W$ of $C$ such that $\alpha(Q)$ is bounded for each $Q \in W \cap A_{\infty}$. Assume to the contrary that there exists a sequence $Q_{i} \in A_{\infty}$ tending to a point $P \in C$ with $\alpha\left(Q_{i}\right)$ all unbounded. Then, by the continuous dependence of solutions on initial conditions, for each $R>0$ large enough we can choose a sequence $t_{i}<0$ such that $\left\|x\left(t_{i}, P_{i}\right)\right\|=R$ and $x\left(t_{i}, P_{i}\right)$ tends to a point $P^{*} \in A_{\infty}$ as 
$i \rightarrow \infty$. In view of Olech's Lemma we can take a neighbourhood $O$ of $P^{*}$ so that $P_{i} \in\{x(t, Q): Q \in O, t>0\}$ for $i$ large enough. This implies that $P \in O \subset A_{\infty}$, contrary to our assumptions. Thus a neighbourhood $W$ as above does exist. By the same argument, we can also prove that $\alpha(Q) \subset C$ for all $Q \in W$. So, each connected component of $\mathrm{Bd} A_{\infty}$ can be viewed as a repulsor of $(\mathrm{S})$.

Now, we prove the connectedness of $\mathrm{Bd} A_{\infty}$. Assume to the contrary that $\mathrm{Bd} A_{\infty}$ has some connected components $c_{i}, i=1,2, \ldots$ For each $c_{i}$ we denote by $R_{c_{i}}$ the set of all points $P$ lying outside $c_{i}$ such that $\alpha(P)=c_{i}$. Clearly, $R_{c_{i}}$ is a non-empty open subset of $A_{\infty}$ and $\mathrm{Bd} R_{c_{i}} \cap A_{\infty} \neq \emptyset$. Fix $c=c_{1}$, let $P \in \operatorname{Bd} R_{c_{1}} \cap A_{\infty}$ and let $L$ be the trajectory of ( $\left.\mathrm{S}^{*}\right)$ through $P$. If the trajectory $x(t, P)$ crosses $L$ once again, then $\alpha(P)$ is bounded, and consequently $\alpha(P)=c_{i}$ for some $i \neq 1$. This is impossible, since $P \in \operatorname{Bd} R_{c_{1}}$. Thus, as in the proof of Lemma 2, no solution curve starting from $L$ crosses $L$ again. This implies that $L \cap R_{c_{1}}$ is a closed subset of $R_{c_{1}}$. Now, we take a segment $P Q$ of $L$ such that $Q \in R_{c_{1}}$, and $P^{\prime} \in P Q \backslash\{P, Q\}$. Put

$$
\begin{gathered}
U:=\left\{x\left(t, Q^{\prime}\right): Q^{\prime} \in P^{\prime} P, t \in \mathbb{R}\right\}, \\
V:=R_{c_{1}} \backslash\left\{x\left(t, P^{\prime}\right): t \in \mathbb{R}\right\} .
\end{gathered}
$$

Clearly, $U$ is a non-empty open connected proper subset of $V$ and $V$ is an open connected set. This implies that $\operatorname{Bd} U \cap V \neq \emptyset$. Consequently, there is a $P^{*} \in V \cap \operatorname{Bd} U$ such that the curve $\left\{x\left(t, P^{*}\right): t>0\right\}$ does not intersect the segment $P^{\prime} P$. Applying Olech's Lemma we can take a neighbourhood $O$ of $P^{*}$ such that the solution curves starting from $O$ do not cross the segment $P^{\prime} P$. This contradicts the definition of $U$, since $P^{*} \in \operatorname{Bd} U \cap V$. Hence, we get the contradiction that $\mathrm{Bd} R_{c_{1}} \cap A_{\infty}=\emptyset$.

Thus $\mathrm{Bd} A_{\infty}$ is connected and contains all negative limit sets of all points $P \in A_{\infty}$. Let $P \in A_{\infty}$. In view of the Poincaré-Bendixson Theorem $\alpha(P)$ is either a point, a closed regular cycle or a polycycle. Since we have assumed that case (a) does not hold, in the first case $\alpha(P)$ is a repulsive point. One can verify that in the remaining cases $\alpha(P)=\mathrm{Bd} A_{\infty}$. The proof is complete.

Notice that in case (b) of Lemma 5, infinity can be viewed as an "attracting point" of $(\mathrm{S})$.

Lemma 5. Assume that the conditions (7)-(10) hold. Then case (b) of Lemma 4 cannot happen.

P r o of. Suppose that case (b) of Lemma 4 holds. This means that $A_{\infty}$ is a neighbourhood of infinity, $\mathrm{Bd} A_{\infty}$ is a repulsive point, a limit cycle or a complex limit cycle, and for each $P \in A_{\infty}, \alpha(P)=\operatorname{Bd} A_{\infty}$. Define $c:=\operatorname{Bd} A_{\infty}$. 
First, we consider the case when $c$ is a cycle or a polycycle. Since $c$ is the $\alpha$-limit set of each point in $A_{\infty}$, we can choose a point $P \in A_{\infty}$ close enough to $c$ so that the trajectory $x(t, P)$ crosses the trajectory $x^{*}(t, P)$ more than once. Therefore, in view of Lemma $2, x(t, P)$ crosses $x^{*}(t, P)$ infinitely many times. Let $\left\{t_{i}\right\}_{i=0,1, \ldots,}, 0=t_{0}<t_{1}<\ldots$, be such that $x\left(t_{i}, P\right)$ lies on the trajectory $x^{*}(t, P)$. Let $\gamma_{i}$ be the closed curve composed by the segment $L_{i}:=x\left(t_{i-1}, P\right) x\left(t_{i}, P\right)$ on the trajectory $x(t, P)$ and $L_{i}^{*}:=$ $x^{*}\left(t_{i-1}, P\right) x^{*}\left(t_{i}, P\right)$ on the trajectory $x^{*}(t, P)$, and let $D_{i}$ be the bounded component of $\mathbb{R}^{2} \backslash \gamma_{i}$. In view of Olech's Lemma and Remark 1 one can see that $\mathbb{R}^{2}=\bigcup_{i} D_{i}$. By Green's formula we have

$$
0<\int_{L_{i}^{*}}\left\|f\left(x^{*}(t, P)\right)\right\|^{2} d x=\int_{D_{i}} \operatorname{Div} f(x) d x .
$$

So, taking $i \rightarrow \infty$, we get

$$
0 \leq \int_{\mathbb{R}^{2}} \operatorname{Div} f(x) d x
$$

which is impossible by $(10)$.

Now, we consider the case when $c$ is a repulsive point. In this case $A_{\infty}$ is the whole of $\mathbb{R}^{2} \backslash c$. For each circle $S_{r}:=\{x:\|x\|=r\}$ and $\varepsilon>0$ small enough we can take a closed curve $\gamma_{\varepsilon}(r)$ composed of a finite number of solution segments $P_{i} P_{i+1}$ of $(\mathrm{S})$ and $P_{i} Q_{i}$ of $\left(\mathrm{S}^{*}\right), i=1, \ldots, n$, so that

(a) The corresponding functions $P_{i}(t), Q_{i}(t)$ and $G_{i}(t)$ are well defined and the properties (i), (ii), (iii)' of Remark 1 and (4) hold.

(b) $\gamma_{\varepsilon}(r) \subset\{x: r-\varepsilon<\|x\|<r+\varepsilon\}$.

Then, for such closed $\gamma_{\varepsilon}(r)$, from (1) and (4) it follows that

$$
2 \pi r \max _{\|x\| \leq r+\varepsilon}\|f(x)\|^{2} \geq \sum_{i} L\left(P_{i} Q_{i}\right)>-\int_{\mathbb{R}^{2} \backslash D_{\gamma_{\varepsilon(r)}}} \operatorname{Div} f(x) d x .
$$

Now, taking $\varepsilon \rightarrow 0$ and $r \rightarrow 0$ we get

$$
0 \geq-\int_{\mathbb{R}^{2}} \operatorname{Div} f(x) d x,
$$

which is impossible by (10). The proof is complete.

Proof of Theorem 1. If $A_{\infty} \neq \emptyset$, then in view of Lemmas 4 and 5 there is a $P$ such that $\omega(P)=\emptyset$ and $\alpha(P)$ is a saddle point. Assume that $A_{\infty}=\emptyset$. In view of Lemma 4 either (S) has a global attractor or there is a neighbourhood of infinity filled up with closed trajectories of $(\mathrm{S})$. However, 
for each closed trajectory $\gamma$ of $(\mathrm{S})$,

$$
\int_{\gamma} \operatorname{Div} f(x) d x=0
$$

Hence, by condition (iv) the last case is impossible. Thus, the system (S) has a global attractor.

Remark 2. As in the proof of Theorem 1 and of the above lemmas, under conditions (i)-(iii) of Theorem 1 either the structure of the system (S) has one of types (a), (b) or (c) of Lemma 3, or infinity can be viewed as an "attracting point" of (S).

For polynomial systems, we obtain

THEOREM 2. Let $f$ be a polynomial map of $\mathbb{R}^{2}$ into itself such that conditions (i)-(iii) of Theorem 1 hold. Then either (a) or (b) of Theorem 1 holds for $(\mathrm{S})$, or

(c) every trajectory of $(\mathrm{S})$ is either a centre, a saddle point, a closed curve or a curve joining two saddle points.

Proof. Consider the system (S) when $f$ is a polynomial. In this case, by (iii) the polynomial function $\operatorname{Div} f(x)$ is non-positive outside a bounded set. One can verify that either $\operatorname{Div} f \equiv 0$ or

$$
\int_{\mathbb{R}^{2}} \operatorname{Div} f(x) d x=-\infty .
$$

For the second case, in view of Theorem 1 either (S) has a global attractor or there is a point $P$ such that $\omega(P)=\emptyset$ and $\alpha(P)$ is a saddle point of $(\mathrm{S})$.

Now, assume that $\operatorname{Div} f \equiv 0$ and there is no $P$ such that $\omega(P)=\emptyset$ and $\alpha(P)$ is a saddle point. Since Div $f \equiv 0$ the singular points of $(\mathrm{S})$ are centres or saddle points and $(\mathrm{S})$ has no limit cycle and no complex limit cycle. Furthermore, in view of Lemma 4 the set $A_{\infty}$ is empty and there is a neighbourhood of infinity filled up with closed trajectories. From these facts it follows that every trajectory of $(\mathrm{S})$ is either a centre, a saddle point, a closed curve or a curve joining two saddle points.

4. Univalence domain of a map. In this section we apply Theorems 1 and 2 to the uniqueness problem for the equation

$$
f(x)=a .
$$

A special case of Theorem 1 is when the system (S) has no saddle point. In this case the behavior of the system at infinity is simple. 
Set

$$
\begin{aligned}
I_{f}:=\left\{a \in \mathbb{R}^{2}: 0<\# f^{-1}(a)\right. & <\infty \text { and } \\
& \left.\operatorname{det} D f(x)>0 \text { for all } x \in f^{-1}(a)\right\} .
\end{aligned}
$$

The method of proof of Theorem 1 allows us to obtain

THEOREM 3. Suppose that the interior int $I_{f}$ of $I_{f}$ is non-empty and that condition (ii) of Theorem 1 holds. Then the map $f: f^{-1}\left(\operatorname{int} I_{f}\right) \rightarrow \operatorname{int} I_{f}$ is bijective.

Proof. Assume that int $I_{f} \neq \emptyset$.

Step 1. We prove that there exists an open dense subset $U$ of int $I_{f}$ such that for each $a \in U$,

$$
\|f(x)-a\|>\text { const }>0 \quad \text { for }\|x\|>\text { const. }
$$

Let $N: \operatorname{int} I_{f} \rightarrow \mathbb{Z}$ be defined by $N(a)=\# f^{-1}(a)$. By the definition of $I_{f}$, the restriction of $f$ to int $I_{f}$ is a local diffeomorphism. Hence, $N$ is lower-semicontinuous on int $I_{f}$. Put $V_{k}:=\{a: N(a) \leq k\}$ for non-negative integers $k$. Then each $V_{k}$ is a closed subset of int $I_{f}$. Put $U_{k}:=\operatorname{int}\left(V_{k} \backslash V_{k-1}\right)$ and $U:=\bigcup_{k} U_{k}$. Then, by the Baire Category Theorem, $U$ is an open dense subset of int $I_{f}$. One can verify that every $a \in U$ satisfies (22).

Step 2. Consider the system

$$
\dot{x}=f(x)-a
$$

for each $a \in U$. Then the systems $\left(\mathrm{S}_{a}\right)$ satisfy conditions (i)-(iii) of Theorem 1. Furthermore, by definition $\left(\mathrm{S}_{a}\right)$ has only stable singular points. As pointed out in Remark 2 , in this case infinity can be viewed as a repulsive, attracting or centre point of $\left(\mathrm{S}_{a}\right)$. We now show that $\left(\mathrm{S}_{a}\right)$ has a unique singular point.

Let $P$ be a singular point of $\left(\mathrm{S}_{a}\right), f(P)=a$. Denote by $D_{P}$ the stable domain of $P$, which is a repulsive, attracting or centre domain of $P$ according to the singular type of $P$. Applying Lemmas 1, 3 and 4 we see that either $\operatorname{Bd} D_{P} \backslash\{P\}$ is a closed trajectory or $D_{P}$ is the whole of $\mathbb{R}^{2} \backslash\{P\}$. In the last case, $P$ is a unique singular point of $\left(\mathrm{S}_{a}\right)$.

Assume that for each singular point $P$ of $\left(\mathrm{S}_{a}\right)$ the set $\mathrm{Bd} D_{P} \backslash\{P\}$ is a closed trajectory. Then the boundary of the stable domain of infinity is also a closed trajectory and all singular points lie inside this closed trajectory. So, by the well-known fact that inside a closed trajectory there is at most one stable singular point or there is a saddle point, the point $P$ is the unique singular point of $(\mathrm{S})$.

Thus, we see that $N \equiv 1$ on $U$. Since $f$ is a local diffeomorphism on $f^{-1}\left(\operatorname{int} I_{f}\right)$ and $U$ is an open dense subset of $\operatorname{int} I_{f}$, we have $N \equiv 1$ on $\operatorname{int} I_{f}$. This means that $f: f^{-1}\left(\operatorname{int} I_{f}\right) \rightarrow \operatorname{int} I_{f}$ is bijective. 
This theorem together with a result of Newman [6], which asserts that every injective polynomial map from $\mathbb{R}^{n}$ to itself is necessarily bijective, allows us to obtain immediately the following consequence, which gives a contribution to the real version of the Jacobian Conjecture: every polynomial map of $\mathbb{R}^{n}$ into itself without a singularity is a diffeomorphism of $\mathbb{R}^{n}$ (see e.g. $[2,4,9])$.

THEOREM 4. Suppose that $f$ is a polynomial map of $\mathbb{R}^{2}$ into itself such that $\operatorname{det} D f>0$ on $\mathbb{R}^{2}$, except possibly on a finite set. Assume that condition (ii) of Theorem 1 holds. Then $f$ is a homeomorphism of $\mathbb{R}^{2}$.

Let us end the paper by the following example.

EXAMPLE. Consider the 1-parameter family of maps

$$
f_{\mu}\left(x_{1}, x_{2}\right)=\left(x_{2}-x_{1}\left(x_{1}^{2}+x_{2}^{2}-\mu\right),-x_{1}-x_{2}\left(x_{1}^{2}+x_{2}^{2}-\mu\right)\right)
$$

with $\mu \in \mathbb{R}$ and the systems

$$
\dot{x}=f_{\mu}(x) .
$$

For this family

$$
\begin{gathered}
f_{\mu}^{-1}(0)=\{0\} \\
\operatorname{det} D f_{\mu}\left(x_{1}, x_{2}\right)=3\left(x_{1}^{2}+x_{2}^{2}\right)^{2}-4 \mu\left(x_{1}^{2}+x_{2}^{2}\right)+\mu^{2}+1, \\
\operatorname{Div} D f_{\mu}\left(x_{1}, x_{2}\right)=2 \mu-4\left(x_{1}^{2}+x_{2}^{2}\right)
\end{gathered}
$$

We have

(1) For $\mu \leq 0$, the system $\left(\mathrm{S}_{\mu}\right)$ has the structural type (a) and $f_{\mu}$ is a diffeomorphism of $\mathbb{R}^{2}$.

(2) For $0<\mu<3^{1 / 2}, f_{\mu}$ is a diffeomorphism of $\mathbb{R}^{2}$ and the system $\left(\mathrm{S}_{\mu}\right)$ is of type (b).

(3) For $\mu=3^{1 / 2}, f_{\mu}$ is a homeomorphism of $\mathbb{R}^{2}$ with a unique singularity at 0 and the system $\left(\mathrm{S}_{\mu}\right)$ is of type $(\mathrm{b})$.

(4) For $\mu>3^{1 / 2}$, the system $\left(\mathrm{S}_{\mu}\right)$ is also of type (b), but $f_{\mu}$ is not a diffeomorphism of $\mathbb{R}^{2}$. However, $I_{f_{\mu}}$ is a non-empty open set and $0 \in \operatorname{int} I_{f_{\mu}}$.

\section{References}

[1] V. I. Arnold and Yu. S. Il'yashenko, Ordinary differential equations. I, in: Dynamical Systems I, Ordinary Differential Equations and Smooth Dynamical Systems, D. V. Anosov and V. I. Arnold (eds.), Springer, New York, 1988, 7-149.

[2] H. Bass, E. F. Connell and D. Wright, The Jacobian Conjecture, Bull. Amer. Math. Soc. 7 (1982), 287-330.

[3] A. Białynicki-Birula and M. Rosenlicht, Injective morphisms of algebraic varieties, Proc. Amer. Math. Soc. 13 (1962), 200-204.

[4] O. H. Keller, Ganze Cremona-Transformationen, Monatsh. Math. Phys. 47 (1939), 299-306. 
[5] L. Markus and H. Yamabe, Global stability criteria for differential systems, Osaka Math. J. 12 (1960), 305-317.

[6] D. J. Newman, One-one polynomial maps, Proc. Amer. Math. Soc. 11 (1960), 867870.

[7] C. Olech, On the global stability of an autonomous system on the plane, in: Contributions to Differential Equations 1 (1963), 389-400.

[8] -, Global phase-portrait of a plane autonomous system, Ann. Inst. Fourier (Grenoble) 14 (1964), 87-98.

[9] C. Olech and M. Meisters, Solution of the global asymptotic stability Jacobian Conjecture for the polynomial case, in: Analyse Mathématique et Applications, Gauthier-Villars, Paris, 1988, 373-381.

INSTITUTE OF MATHEMATICS, HANOI

P.O. BOX 631

10000 BOHO, HANOI, VIETNAM

Reçu par la Rédaction le 20.10.1994 